\title{
Decision Model Applied in IoT for Green Buildings Based on Grey Incidence Analysis and ANN
}

\author{
Liping Wang $\mathbb{D}^{D}$, Dongyao Zou, Yanpei Liu, and Guangyong Xi \\ School of Computer and Communication Engineering, Zhengzhou University of Light Industry, Zhengzhou 450000, China \\ Correspondence should be addressed to Liping Wang; 16432562@qq.com
}

Received 11 June 2021; Revised 18 December 2021; Accepted 3 January 2022; Published 8 February 2022

Academic Editor: Vishal Sharma

Copyright (c) 2022 Liping Wang et al. This is an open access article distributed under the Creative Commons Attribution License, which permits unrestricted use, distribution, and reproduction in any medium, provided the original work is properly cited.

\begin{abstract}
Due to the fewer uncertainty samples and information-lacking problems in the decision-making center of the Internet of Things (IoT) for green buildings, the optimized model was selected as the preferred method in settlement prediction. In this paper, we proposed the adaptive adjustment strategies of the application layer in IoT for green buildings based on grey incidence analysis and artificial neural networks (ANNs). An additional layer $\mathrm{H}$ fuzzy propagation natural network algorithm was introduced to collect sensing layer data of IoT and adaptively adjust decisions. The energy-saving control of the building needs to be adjusted continuously; therefore, we have taken a grey incidence evaluation to obtain adjustment of the parameters. At the same time, the actual Heating Ventilation Air Conditioning subsystem is often in the grey state above, and the current control system of its system is missing the corresponding adjustment scheme. The introduction of the data evaluation in the data center for adaptive adjustment of input data is an effective solution. The real-time running result shows that the proposed solution reduces energy consumption by over $30 \%$ compared to the state-of-the-art approaches while having on average $10 \%$ fewer expired measurements. The strategies have a significant impact on energy savings for green buildings.
\end{abstract}

\section{Introduction}

The operation of a green building using IoT focuses on energy savings, but the lack of data has led to most actual building energy consumption savings without improving energy efficiency. A survey of the actual efficiency of 156 certified buildings in the United States by the US Green Building Council (USGBC) found that the energy and climate environment, as well as the indoor environment of buildings, were above standard. Green building itself is not static; the aging of materials, equipment replacement, and so on are the cause of changes in control conditions; good operation needs to be constantly adjusted according to the state of the building and the change of climate $[1,2]$. On the other hand, with the progress of science and technology in recent decades, the widespread use of green technology, and the widespread use of green building materials, material costs are also significantly reduced, and the inherent energysaving technology has been seriously challenged [3]. The deployment of IoT applications in green buildings inevitably generates large-scale data sets on the operating status of the building, including the area temperature, air quality, user distribution, and environmental illumination $[4,5]$. The key issues are how to use the network data to control the operation of the entire building, supervise abnormal status, and reduce energy consumption as much as possible while ensuring the comfort of users. The related works are mainly based on specific applications, but the data collection and fusion of the sensory layers are short of the analysis of building operation characteristics and strategies. In addition, as the complexity of the building system, data usually comes from various types of sensor platforms deployed in different locations and collected in the network data center. Thus, it is necessary to use data fusion technology to establish internal correlations with multisource sensor data collected through the network while improving the system's cognitive ability.

The decision-making fusion of sensory layers based on intelligent algorithms can realize the identification and optimization of building system behaviour while maintaining user comfort experience. Mendhe et al. used artificial 
neural network (ANN), fuzzy logic (FL) modelling, and evolutionary algorithms in intelligent computing to mine and process building sensor network data to make building systems more effective in dynamic perception and optimization [6, 7]. In some studies, the ANN technology is used to complete dynamic prediction and overall modelling of the system, and it can also be adaptively adjusted according to new requirements through online learning [8-10]. While using ANN technology to extract and model system behaviour, fuzzy logic technology is usually used to quantify and model nondeterministic events, which can ensure system adaptive adjustment in the presence of noise and interference with unreliable data $[11,12]$. Rawat et al. used fuzzy clustering-neural network (FC-ANN) technology to fuse multisource data from sensor networks to provide realtime monitoring and analysis of building operation status [13]. Rawat et al. also combined rough set and ANN technology in the multisensor data fusion of the sensory layer of IoT for buildings to improve the accuracy of network behaviour prediction $[14,15]$. Metallidou et al. proposed an intelligent building template that manages the performance of all technology systems through IoT technologies for energy efficiency [16]. Ivan et al. modelled the work on the data collected by IoT devices, studied the superparameter selection of the proposed lost data recovery technology, and improved the accuracy of the work [17]. Since most of the processing of sensory layer information in current green buildings is mainly to monitor terminals, it often requires the network data center to have an information processing plan for monitoring and executing decisions. Also, due to the multisources of network perception information and the variability of environmental factors, we considered the use of artificial intelligence algorithms in the data center while taking into account the online learning and adaptive adjustment capabilities of the control decision algorithm.

The evaluation of the applicability of related technologies throughout the life cycle of buildings and the modification of the suitability of existing buildings can be done through diagnostic and control systems based on IOTs. The traditional PID mode is easy to cause system fluctuations, leading to frequent adjustments and reducing system robustness. In recent years, the fuzzy neural network, which is widely used, has the characteristics of self-learning and association and can use the existing experience knowledge to process and describe fuzzy information, which has great advantages in dealing with nonlinearity, fuzziness, and other problems. But it also faces the following shortcomings. The first is the uncertainty of the sample. In the actual process, debugging values are used as training samples for neural networks, but the actual information is often uncertain and it is impossible to define whether they are optimal values. Second, samples in all modes cannot be collected during system commissioning and training. In the case of HVAC (Heating Ventilation Air Conditioning) system, climate factors affect their working status. If both are actually commissioned and trained, they need to go through seasonal changes. Therefore, it is difficult to obtain the training set. Finally, there are too many repeated training sessions. Because of its own aging or equipment update, personnel behaviour changes, and the sample space needs to be updated and constantly trained. However, overtraining will lead to a reduction in generation capacity. Our research put forward the H-FBPNN (Fuzzy Back Propagation Natural Network) algorithm model based on grey incidence analysis in the data center of IoT used in green buildings, applies the adaptive energy-saving adjustment to HVAC system, and verifies the process of adaptive adjustment of the corresponding parameters in the operation of the proposed algorithm model by the project example and the resulting energy-saving effect.

\section{H-FBPN Algorithm Model}

The framework of distributed decision based on IoT for green buildings is shown in Figure 1 and consists of three layers: the application layer, the compute layer, and the sensing layer. The compute layer collects all the information from the sensing layer and analyses it, consisting of two modules: the algorithm module and the database module. The database provides all real-time and eventdriven data for the algorithm module, including energy usage comparisons, historical data analysis, and pattern matching. The analysis tools used by the algorithm module are selected by the application layer as needed. For example, the HVAC subsystem has large dynamic inertia. The control quantity is temperature, carbon dioxide concentration, etc., usually with a long response time. Therefore, traditional sensor network scheme has pure hysteretic. In addition to its nonlinear characteristics, it is difficult to carry out accurate mathematical modelling [18]. In the lighting subsystem, the illumination sensor is greatly affected by the environment, and the traditional PID mode is easy to cause system fluctuations, resulting in frequent adjustment and reduction of the robustness of the system [19].

We proposed the decision model of the fuzzy neural network-attached $\mathrm{H}$ computing layer, in which the backpropagation (BP) algorithm was applied to train neural network. The principle of attaching $\mathrm{H}$ operation layer is that the environment sequence $E(t)$ and the system output $S(t)$ are quantified and evaluated continuously. As shown in Figure 2, the additional H layer-fuzzy BP neural network (H-FBPNN) is used for the control decision center, which consists of the fuzzy layer, $\mathrm{H}$ layer, impact evaluation layer, BP input layer, implied layer, and output layer. The detailed description is described as follows:

(i) Fuzzy layer: according to the set fuzzy membership function, the sequence of ambient quantity collected by the input network generates a dimensionless vector of $\mu(E(t))$.

(ii) $\mathrm{H}$ layer: the decision center outputs the control signal C $(t)$ at $t$ and gets the time series of the system dynamic variable $\Delta D$ after the fixed sampling interval $\tau$. The environmental time series $E^{\mathrm{T}}(t)$ and state sequence $S^{\mathrm{T}}(t)$ are obtained at a fixed sampling interval, and the evaluation coefficient $H\left(S^{\mathrm{T}}, E^{\mathrm{T}}\right)$ is 


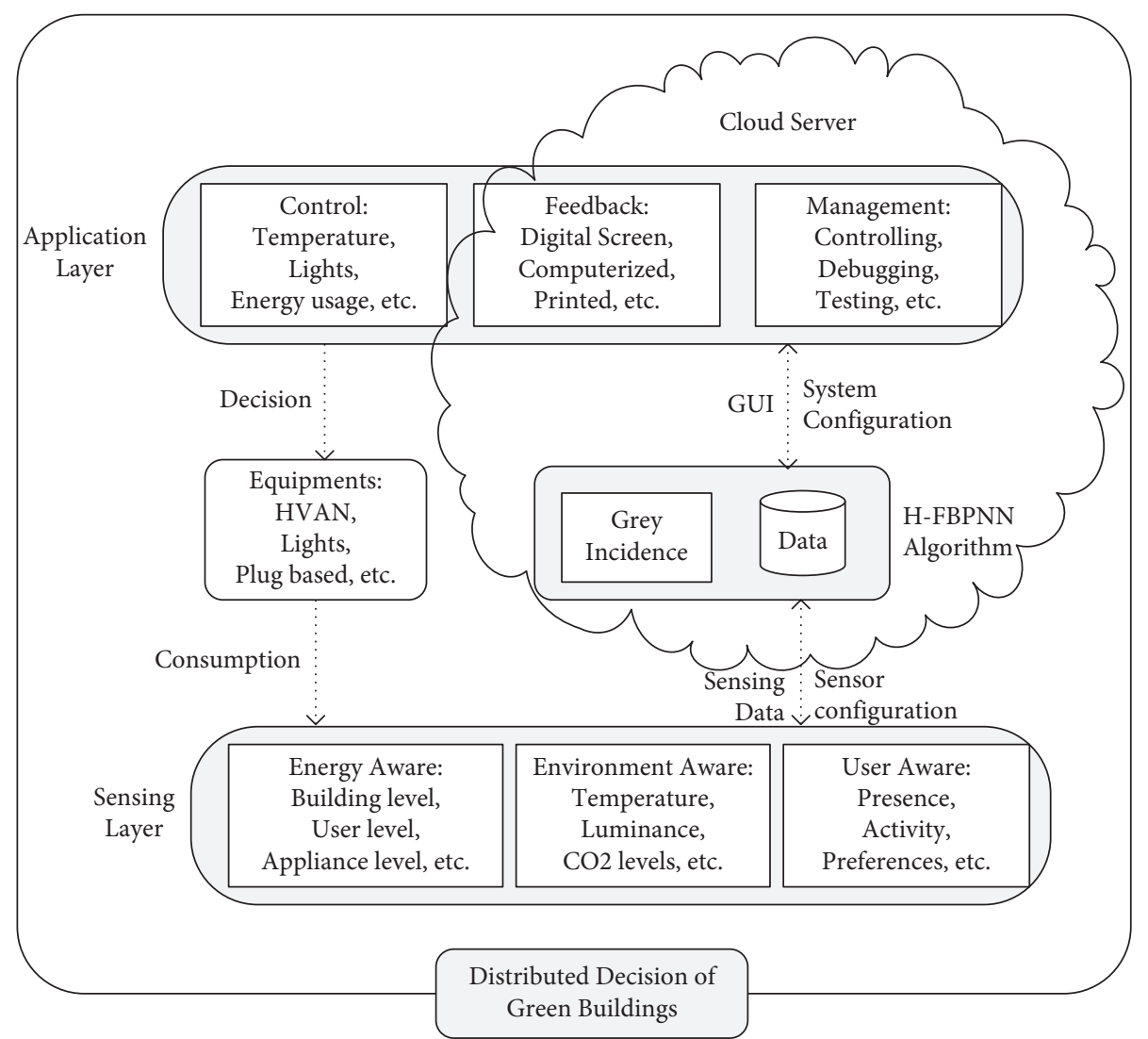

FIGURE 1: Framework of distributed decision based on IoT.

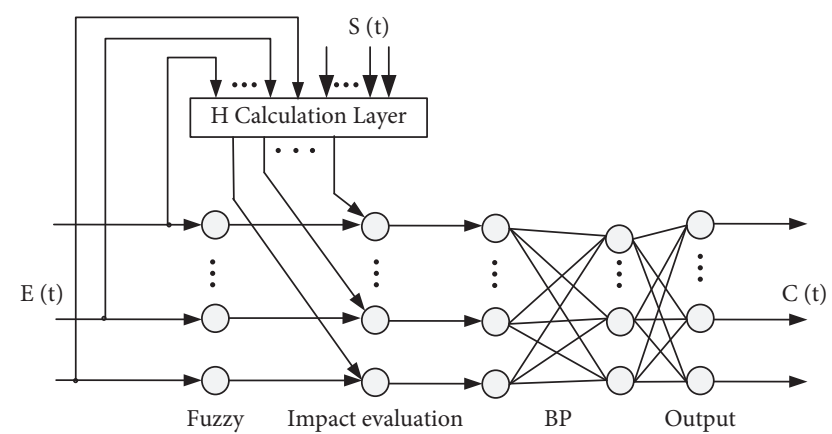

Figure 2: H-FBPNN algorithm model.

generated for the control system through the H layer operation $E^{\mathrm{T}}(t)$ and $S^{\mathrm{T}}(t)$.

(iii) Impact evaluation layer: $\mu(E(t))$ is obtained by the fuzzy generalization of sensing time series $E(t)$ and is multiplied by $H\left(S^{T}, E^{T}\right)$ in the impact evaluation layer to be $I(t)$, such as the following formula:

$$
I(t)=H(S T, E T) . * \mu(E(t)) .
$$

(iv) BP layer is output directly to the implied layer after a combination of $I(t)$ calculations.

(v) Output layer is the output control signal $C(t)$.

The traditional fuzzy BP neural network has an additional $\mathrm{H}$ computing layer, which can be used to evaluate the impact of input variables online and directly make quantitative evaluation gain coefficient $H\left(S^{T}, E^{T}\right)$. The grey correlation analysis theory is applied in the correlation analysis of various environment-perceived variables and state variables in $H$ operation. Thus, the error of judgment caused by the fluctuation of sample data difference and the number of updates of training samples is reduced, which improves the stability of the system.

\section{Grey Incidence Analysis}

We constructed a new type of grey index incidence model, which has the characteristics of parallelism, consistency, and pruning. Besides, that the model has a perfect index for the measurement between sequences is more in line with the theory system of grey incidence. The analysis of grey correlation comes from the grey theory, which was first proposed by Julong Deng of Huazhong University of Science and Technology in 1982 [20]. The basic idea of grey incidence analysis is to determine the correlation degree between the comparison sequences and the reference sequence; therefore, the more similar their trend-lines are, the higher the correlation is. The reference sequence should be an ideal comparison criterion, which can be composed of the optimal value (or the worst value) of each indicator, or other reference values can be selected according to the evaluation purpose. According to the corresponding axiom principle and the relevant theory, the degree of correlation 
between the reference sequence and several comparison series is grey. The traditional grey correlation model cannot satisfy parallelism, consistency, and pruning at the same time [21-23].

3.1. New Grey Incidence Model. The sequence of state time collected within the set time period $d$ is $S=[s(1), s(2), \ldots s(k)$, $\ldots, s(n)]$. Environment variable time series is $E_{i}=\left[e_{\mathrm{i}}(1)\right.$, $\left.e_{\mathrm{i}}(2), \ldots, e_{\mathrm{i}}(k), \ldots, e_{\mathrm{i}}(n)\right](i=1,2, \ldots, m)$. The variable sequence is $X=\left\{S, E_{\mathrm{i}}\right\}$, in which $X_{0}=S, X_{i}=E_{\mathrm{i}}(i=1,2, \ldots, m)$. The association factor is constructed as below.

The input of the $\mathrm{H}$ layer is processed with interval valuebased operator $D_{3} . X_{0}$ and $X_{\mathrm{i}}$ are converted to $Y_{0}$ and $Y_{\mathrm{i}}$, which are nonnegative real sequences by uniformization. Grey operators are applied as follows:

$$
\begin{aligned}
& y_{0}(k)=x_{0}(k) d_{3}=\frac{x_{0}(k)-\min _{k} x_{0}(k)}{\max _{k} x_{0}(k)-\min _{k} x_{0}(k)}, \quad k=1,2, \ldots, n, \\
& y_{i}(k)=x_{i}(k) d_{3}=\frac{x_{i}(k)-\min _{k} x_{i}(k)}{\max _{k} x_{i}(k)-\min _{k} x_{i}(k)}, \quad k=1,2, \ldots, n .
\end{aligned}
$$

The grey index incidence coefficient of the sequence of state dynamic variable and environmental dynamic variables are computed. As defined in (3), the grey incidence coefficient is $\gamma_{\mathrm{ik}}$ :

$\gamma_{i k}=\gamma\left(x_{0}(k), x_{i}(k)\right)=\frac{\exp \left(\dashv y_{0}(k)-y_{i}(k) \mid\right)-\exp (-1)+\theta}{1-\exp (-1)+\theta}$,

where $\theta$ is the resolution factor $(0<\theta<1)$. If $\theta=0.1$, the value range of $y_{0}(k)$ and $y_{i}(k)$ is between 0 and 1 . The $\gamma_{\text {ik }}$ was plotted as Figure 3.

Entropy weight from information theory was utilized for calculating the incidence degree [24], which is a more objective method of empowerment than the general method of averaging grey incidence coefficient $\gamma_{\mathrm{ik}}$. According to (3), information is provided using the system influencers to determine the weight of each factor.

$$
\gamma_{i}=\gamma\left(X_{0}, X_{i}\right)=\sum_{k=1}^{n} \omega_{i k} \gamma_{i k},
$$

where $\omega_{i k}$ is the weight of the $k$-th element in the $i$-th sequence $\left(\sum_{k=1}^{n} \omega_{i k}=1\right)$. Then, defining a map $\phi:=R \longrightarrow P$ is formulated as

$$
\begin{aligned}
P_{i k>0} & =\frac{\omega_{i k} \gamma_{i k}}{\gamma_{i}}, \\
\sum_{k=1}^{n} P_{i k} & =1, \quad \forall k, P_{i k}>0 .
\end{aligned}
$$

In (5), $P_{i k}$ is defined as the distribution density of weighted grey incidence coefficient, and $P_{i}$ equaling $\left\{P_{i k}\right\}$ is grey connotation sequence. The weighted entropy of grey incidence of $X_{i}$ is provided in the following:

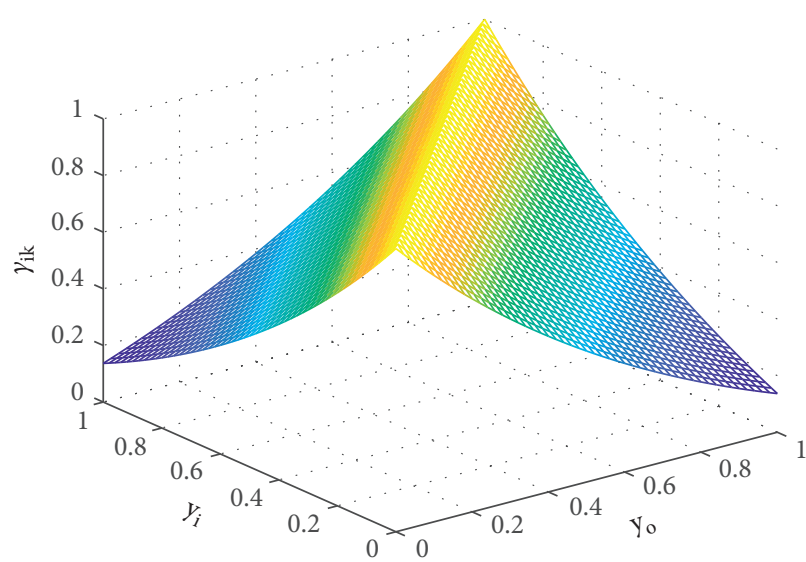

Figure 3: $\gamma_{i k} \operatorname{plot}(\theta=0.1)$.

$$
G\left(\gamma_{i}\right)=-\sum_{k=1}^{n} P_{i k} \ln P_{i k}
$$

The weight $\omega_{i k}(k=1,2, \ldots, m)$ is optimal when the maximum value $G_{\max }$ of $G\left(\gamma_{i}\right)$ is taken. Thus, (5) is turned into a constraint optimization problem:

$$
\begin{aligned}
\operatorname{Max} G\left(\gamma_{i}\right) & =--\sum_{k=1}^{m} P_{i k} \ln P_{i k}, \\
\text { s.t. } P_{i k} & \geq 0 \\
\sum_{k=1}^{m} P_{i k} & =1 \\
\Omega & =\Gamma^{-1} b .
\end{aligned}
$$

In (9),

$$
\begin{aligned}
\Omega_{i} & =\left[\begin{array}{c}
\omega_{i 1} \\
\omega_{i 2} \\
\vdots \\
\omega_{i(n-1)} \\
\omega_{i n}
\end{array}\right], \\
b & =\left[\begin{array}{c}
0 \\
0 \\
\vdots \\
0 \\
1
\end{array}\right], \\
\Gamma & =\left[\begin{array}{ccccc}
\gamma_{i 1} & -\gamma_{i 2} & 0 & \ldots & 0 \\
0 & \gamma_{i 2} & -\gamma_{i 3} & & \vdots \\
\vdots & \ddots & \ddots & \ddots & 0 \\
0 & \ldots & 0 & \gamma_{i(n-1)} & -\gamma_{i n} \\
1 & 1 & 1 & 1 & 1
\end{array}\right] .
\end{aligned}
$$

From (7) to (9), we can see that 


$$
\frac{\omega_{i k} \gamma_{i k}}{\gamma_{i}}=\frac{1}{n}, \quad \forall k, k=1,2, \ldots, n .
$$

The grey incidence analysis model is based on the four axioms of grey incidence. Thus, before using the grey association coefficient constructed in this study on the nonzero sequence $X_{0}$ and $X_{\mathrm{i}}$ after the above conditions, it is necessary to ensure that the constructed grey association coefficient meets the normative, integral, even symmetry, and proximity.

3.2. Quantitative Evaluation of Grey Incidence. The quantitative evaluation of the constructed grey incidence analysis model focus on the parallelism, consistency, affine transformation isotonicity, and convergence of the reference sequence and standard sequence correlation.

At first, the incidence of degree $\gamma\left(X_{0}, X_{i}\right)$ of reference sequences $X_{0}$ and comparison sequences $X_{i}$ must satisfy parallelism. There is a constant named const making (12) set up; thus it is parallel between $X_{0}$ and $X_{i}$. Equation (13) is clearly established, which proves the parallelism of $\gamma\left(X_{0}, X_{i}\right)$.

$$
\begin{gathered}
\forall k, 1 \leq k \leq n \\
\text { s.t. } x_{i}(k)=x_{i}(k)+\text { const, } \\
y_{0}(k)=y_{i}(k) .
\end{gathered}
$$

Secondly, the incidence degree $\gamma\left(X_{0}, X_{i}\right)$ meets consistency, which is proved as follows:

$$
\begin{aligned}
\forall k, 1 & \leq k \leq n \\
\text { s.t. } x_{i}(k) & =x_{i}(k)+\text { const } \\
& \Rightarrow \gamma\left(X_{0}, X_{i}\right)=1, \\
y_{0}(k) & =y_{i}(k) .
\end{aligned}
$$

Thirdly, according to the reference sequences $X_{0}$, if any comparison sequences $X_{t}$ and $X_{l}$ can be taken as the parallel sequences $X_{t}^{\prime}$ and $X_{l}^{\prime}$ as (15) $\sim(17)$, the incidence degree $\gamma\left(X_{0}, X_{i}\right)$ meets affine transformation isotonicity. In the equations, $\alpha_{1} \alpha_{2}, c_{1}$, and $c_{2}$ are constants.

$$
\begin{aligned}
& X_{t}^{\prime}=\alpha_{1} X_{t}+c_{1}, \\
& \alpha_{1} \neq 0, \\
& X_{l}^{\prime}=\alpha_{2} X_{l}+c_{2}, \\
& \alpha_{2} \neq 0,
\end{aligned}
$$$$
\gamma\left(X_{0}, X_{t}\right)<\gamma\left(X_{0}, X_{l}\right) \Rightarrow \gamma\left(X_{0}, X_{t}^{\prime}\right)<\gamma\left(X_{0}, X_{l}^{\prime}\right) .
$$

Therefore, (17) has been proven to be as follows. Equation (18) can be obtained through the parallel sequences $X_{t}^{\prime}$

$$
\begin{aligned}
\forall k, 1 \leq k \leq n & \\
\text { s.t. } Y_{t}^{\prime}(k) & =\frac{\alpha_{1} x(k)+c_{1}-\min _{k}\left(\alpha_{1} x_{t}(k)+c_{1}\right)}{\max _{k}\left(\alpha_{1} x_{t}(k)+c_{1}\right)-\min _{k}\left(\alpha_{1} x_{t}(k)+c_{1}\right)}=\frac{x_{i}(k)-\min _{k} x_{i}(k)}{\max _{k} x_{i}(k)-\min _{k} x_{i}(k)} \\
& \Rightarrow y_{t}^{\prime}(k)=y_{t}(k) .
\end{aligned}
$$

Finally, the incidence degree $\gamma_{i}$ should be convergent. The reference sequence $X_{0}^{n+T}$ consists of two sequences $X_{0}^{n}$ and $X_{0}^{T}$ which have the elements with the number of $n$ and T. Other than that, the comparison sequence $X_{i}^{n+T}$ consists of a sequence $X_{i}^{n}$ with an element number of $n$ and a sequence $X_{i}^{T}$ with an element number of T. If the incidence coefficient is recorded as $\gamma_{i}^{n}, \gamma_{i}^{T}$, and $\gamma_{i}^{n+T}$, and then by (11) we can get as follows:

$$
\begin{gathered}
\omega_{k}^{n} \gamma_{i k}=\frac{\gamma_{i}^{n}}{n}, \quad k=1,2, \ldots, n, \\
\omega_{k}^{T} \gamma_{i k}=\frac{\gamma_{i}^{T}}{T}, \quad k=n+1, n+2, \ldots, n+T, \\
\omega_{k}^{n+T} \gamma_{i k}=\frac{\gamma_{i}^{n+T}}{n+T}, \quad k=1,2, \ldots, n+T .
\end{gathered}
$$

Evidently,

$$
\begin{aligned}
\gamma_{i}^{n+T} & =(n+T) \omega_{k}^{n+T} \gamma_{i k}, \\
\Rightarrow \gamma_{i k} & =\frac{\gamma_{i}^{n+T}}{\omega_{k}^{n+T}(n+T)}, \\
\frac{\gamma_{i}^{n+T}}{\omega_{k}^{n+T}(n+T)} & =\frac{\gamma_{i}^{n}}{\omega_{k}^{n} n} .
\end{aligned}
$$

According to (20), $\gamma_{i}^{n}$ approximately is equal to $\gamma_{i}^{n+T}$ when $n$ is much bigger than T. Thus, the incidence degree $\gamma_{i}$ is convergent.

\section{Implementation}

We have implemented the proposed model in the HVAC (Heating Ventilation Air Conditioning) subsystem of a big green building, which has obtained LEED Platinum Award. The HVAC subsystem mainly uses the ground-source heat 
pump system. The system supplies air conditioning on the ground floor of the main office building with an area of $6338 \mathrm{~m}^{2}$ and the air conditioning load area of $1068 \mathrm{~m}^{2}$ in plant no. 2. Figure 4 shows the green office building, a total of 4 new air conditioning system, each floor of 2, and new wind unit using a full heat new air exchanger. It has been in operation since October 2017 and has an operating temperature of $26^{\circ} \mathrm{C}$. The working hours are from 9:00 to 17:30, and the equipment runs from 7:00 to 17:30.

4.1. Actual Network Model Building. Based on the H layerfuzzy BP neural network algorithm proposed in this paper, the actual network model is established as follows.

Environment-sensing signals $E(t)$ are collected as (21). Increased energy consumption $P_{l}(t)$ of lighting represents the cooling caused by lighting. The increased energy consumption of $P_{c z}(t)$ and $P_{p g}(t)$ of computer socket work and the fan coil is

$$
\begin{aligned}
E(t) & =\left\{T_{\Delta}(t), P_{l}(t), P_{c z}(t), P_{p g}(t), P_{x}(t)\right\}, \\
T_{\Delta}(t) & =T_{\text {out }}(t)-T_{i}(t) .
\end{aligned}
$$

Control decision has been defined as 8 modes of work as in Table 1, where 0 is for shutdown, $x$ is for contactor closure, motor operation, and inverter frequency conversion, and $50 \mathrm{~Hz}$ is for the nonvariable operating state. The collection of state quantity follows the principle of reducing the transmission point and facing the actual energy consumption level of the bottom layer, using the energy consumption of the pump as the state quantity. The ranges of $11 \mathrm{~kW}$ and $22 \mathrm{~kW}$ variable-frequency pumps are 30 to $50 \mathrm{~Hz}$ so that the output and the state variate-values are equal to the corresponding energy consumption per sampling cycle.

The membership functions for different input variables are defined as follows.

Outdoor membership function was formulated as (22). It is set to turn on air conditioning above $26^{\circ} \mathrm{C}$ and calculate at a maximum temperature of $42^{\circ} \mathrm{C}$.

$$
A_{T_{0}}= \begin{cases}0, & x \leq 26 \\ \frac{x-26}{16}, & 26<x \leq 42 \\ 1, & x>42 .\end{cases}
$$

The lighting membership function is defined as (23) when the sample period is $t_{p}$. The fully open power of the lighting is $5 \mathrm{~kW}$.

$$
A_{l}(x)= \begin{cases}0, & x=0 \\ \frac{x}{5 t_{p}}, & 0<x \leq 5 t_{p}, \\ 1, & x>5 t_{p} .\end{cases}
$$

Socket membership function is defined as (24) when the sample period is $t_{p}$. According to the measured data of the historical database, the maximum reading during the

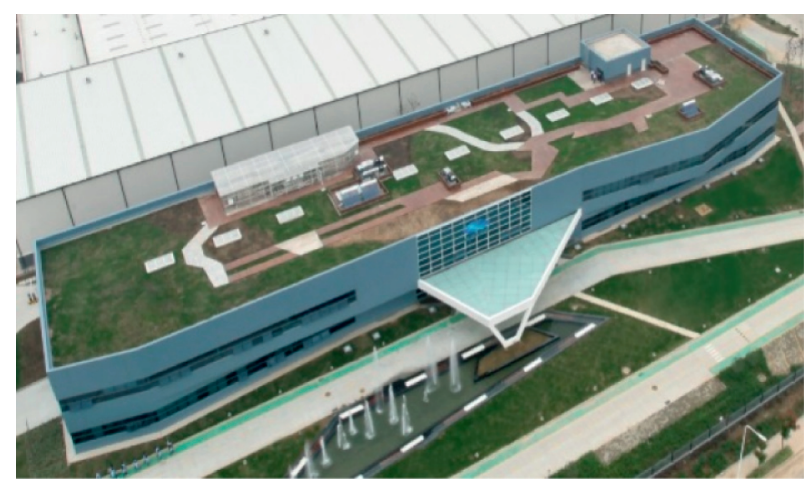

Figure 4: Aerial view of the green office building.

Table 1: Output mode.

\begin{tabular}{lcccccccc}
\hline Mode no. & 1 & 2 & 3 & 4 & 5 & 6 & 7 & $\begin{array}{c}8 \\
(\mathrm{~Hz})\end{array}$ \\
\hline $11 \mathrm{~kW}$ 1\#pump & $\mathrm{X}$ & $50 \mathrm{~Hz}$ & 0 & 0 & $50 \mathrm{~Hz}$ & $50 \mathrm{~Hz}$ & $\mathrm{X}$ & 50 \\
$11 \mathrm{~kW}$ 2\#pump & 0 & 0 & 0 & 0 & 0 & 0 & $50 \mathrm{~Hz}$ & 50 \\
$22 \mathrm{~kW}$ spare & 0 & 0 & $\mathrm{X}$ & $50 \mathrm{HZ}$ & 0 & $50 \mathrm{~Hz}$ & $50 \mathrm{~Hz}$ & 50 \\
pump & 0 & & & &
\end{tabular}

sampling cycle is $4.88 \mathrm{~kW}$, but finally we use $5 \mathrm{~kW}$ for the convenience of calculation.

$$
A_{c z}(x)= \begin{cases}0, & x=0, \\ \frac{x}{5 t_{p},} & 0<x \leq 5 t_{p}, \\ 1, & x>5 t_{p} .\end{cases}
$$

Fan coil membership function is defined as (25) while the sample period is $t_{p}$ and installed power is $37 \mathrm{~kW}$.

$$
A_{c z}(x)= \begin{cases}0, & x=0 \\ \frac{x}{37 t_{p}}, & 0<x \leq 37 t_{p} \\ 1, & x>37 t_{p} .\end{cases}
$$

Air system membership function is defined as (26) while the sample period is $t_{p}$ and installed power is $29 \mathrm{~kW}$.

$$
A_{c z}(x)= \begin{cases}0, & x=0, \\ \frac{x}{29 t_{p},} & 0<x \leq 29 t_{p} \\ 1, & x>29 t_{p} .\end{cases}
$$

4.2. Simulation Analysis. The equipment was debugged with a ten-minute cycle. By sampling the lighting, fan coil, socket, new wind, and the indoor and outdoor temperature 


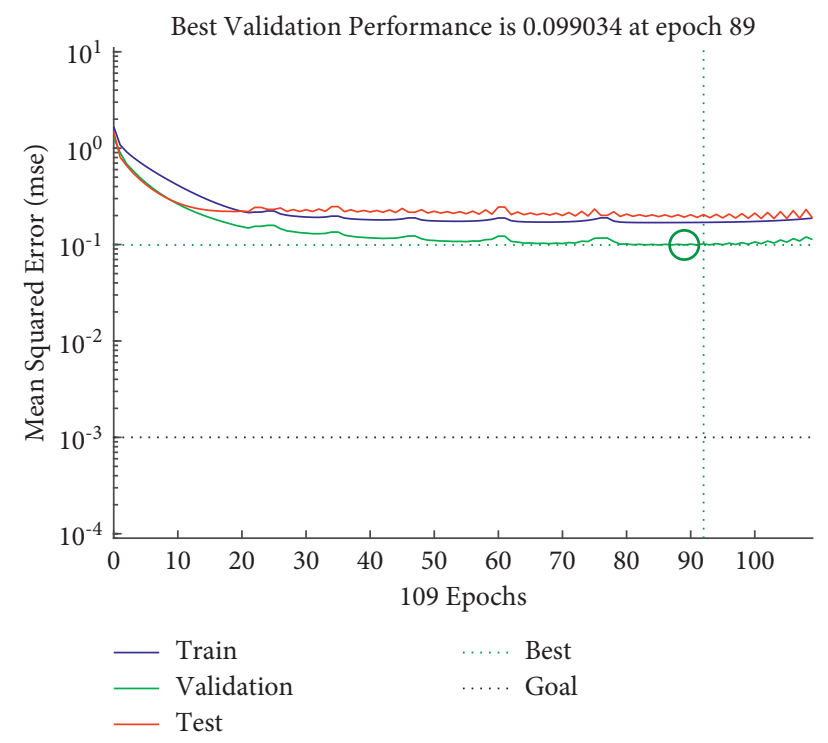

Figure 5: Sample training and verification.

TABLE 2: Factors related to the energy consumption of cold pumps by each input variable.

\begin{tabular}{lccccc}
\hline Temperature range & Lighting & Fan coil & Socket & New air & Data sets \\
\hline $27 \sim 36^{\circ} \mathrm{C}$ & 0.4988 & 0.4923 & 0.2860 & 0.8296 & 0.1244 \\
$22 \sim 35^{\circ} \mathrm{C}$ & 0.2873 & 0.077 & 0.0899 & 0.9706 & 2020.06 .19 \\
$22 \sim 35^{\circ} \mathrm{C}$ & 0.1759 & 0.7647 & 0.3362 & 0.5132 & 2020.07 .13 \\
$20 \sim 30^{\circ} \mathrm{C}$ & 0.1929 & 0.4845 & 0.0124 & 0.123 & 2020.06 .17 \\
$20 \sim 30^{\circ} \mathrm{C}$ & 0.4239 & 0.5275 & 0.3764 & 2020.08 .20 \\
\hline
\end{tabular}

difference is input data, and the energy consumption of the cold water pump is output data. The input layer consists of 5 neurons, the implied layer contains 4 neurons, and the output layer contains an additional $\mathrm{H}$ layerfuzzy BP neural network of 1 neuron. The proposed ANN model is built using Matlab in Windows 10. We selected 1137 sets of data on the condition that the $\mathrm{H}$ layer was shielded, while the training curve is shown in Figure 5. The ratio of training, testing, and evaluation set is 6:2:2. Figure 5 indicates that, due to the uncertainty of the data sample, the training curve fluctuates, affecting the accuracy of the system. Thus, there is still room for further optimization of the model.

We took the resolution factor of 0.0005 and $T$ in days in the grey incidence calculation for the algorithm optimization and then conducted a correlation evaluation and made adjustments accordingly by the $\mathrm{H}$ layer evaluation. Moreover, the data was sampled every 30 minutes from 17:00 to 17:30 during workdays because sometimes the water pump was closed in advance to save energy.

Table 2 shows that the correlation coefficient between the input variables in the training sample and the energy consumption of the cold pump is calculated. The energy consumption of the water pump is the most related coefficient to the energy consumption of new wind. Therefore, in the actual operation, we paid more attention to the management of the new wind opening and took $\eta$ as 0.1 in the effect evaluation for adaptive adjustment.
The curves in Figure 6 represent actual power consumption for a particular workday, with the H-FBPNN model predicting power consumption and the traditional Fuzzy Neural Network Model (FC-NN) predicting power consumption. Obviously, the proposed H-FBPNN model has some improvement in prediction accuracy.

4.3. Performance. According to Figures 7 and 8, the outdoor temperature changes on June 19, 2020, and July 13, 2020, are 22 to 35 degrees Celsius, with the room temperature set to $26^{\circ} \mathrm{C}$, and the system runs from 9:00 to 17:30. The sensor network data center was not used for control decisionmaking on June 19, 2020. After the additional H-Computing Layer-FBPNN algorithm was used on July 13, 2020, the water pump obviously saved $37.84 \%$ more energy than before. As shown in Table 2, the control process is more sensitive to the new wind and temperature under the same weather; then apparently, the effect of fan coils, lighting, and sockets on the water pump is reduced. It is shown that the HVAC cold load in the heat is mainly composed of the imported hot-air by new wind, the building envelope, and the introduced heat by the doors and windows.

According to Figures 9 and 10, the outdoor temperature changes on June 17, 2020, and August 20, 2020, are 20 to 30 degrees Celsius, with the room temperature set to $26^{\circ} \mathrm{C}$, and the system runs from 9:00 to 17:30. The sensor network data center was not used for control decision-making on June 17, 2020. After using the additional $\mathrm{H}$ computing layer FBPN 


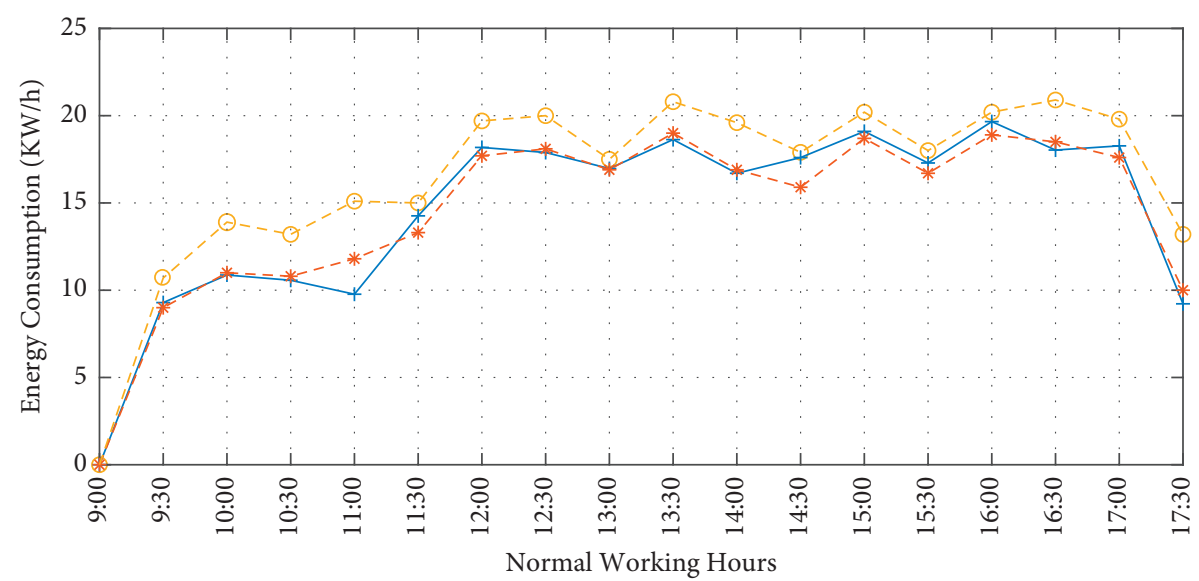

- Actual Power Consumption
$-*-$ H-FBPNN
$-\ominus-$ FC-ANN

FIgURE 6: Comparison of the H-FBPNN model and traditional FC-NN model.

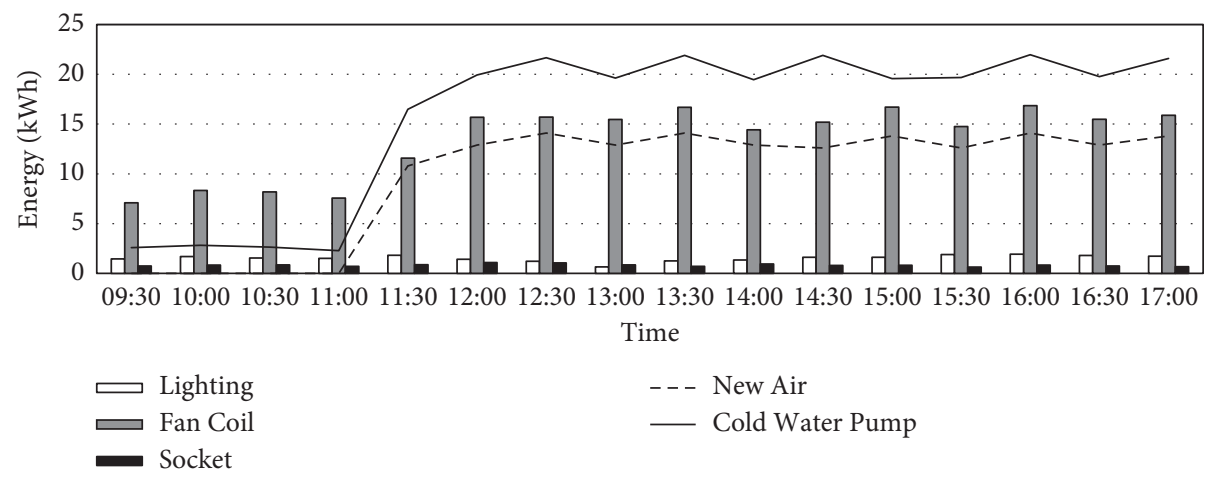

FIGURE 7: Energy consumption in subhour on June 19, 2020.

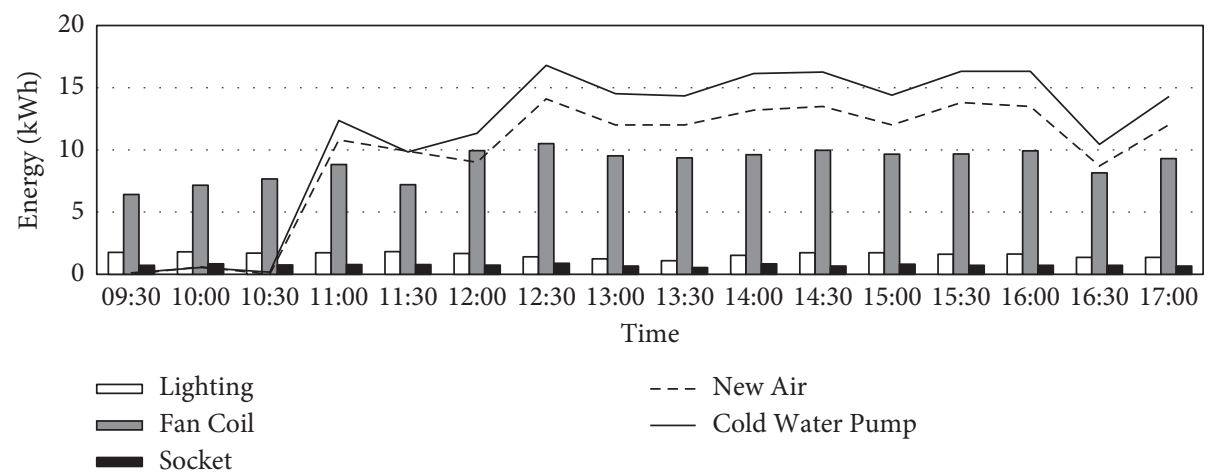

Figure 8: Energy consumption on July 13, 2020.

algorithm to control the decision on July 13, 2020, the cold water pump energy saving was $12.52 \%$, which was significant. The control process is more sensitive to indoor ventilation, lighting, and socket electricity in the same weather, which significantly reduces the effect of the new wind. This also shows HVAC cold load in nonhigh temperature environment mainly in human activity generated by heat. 


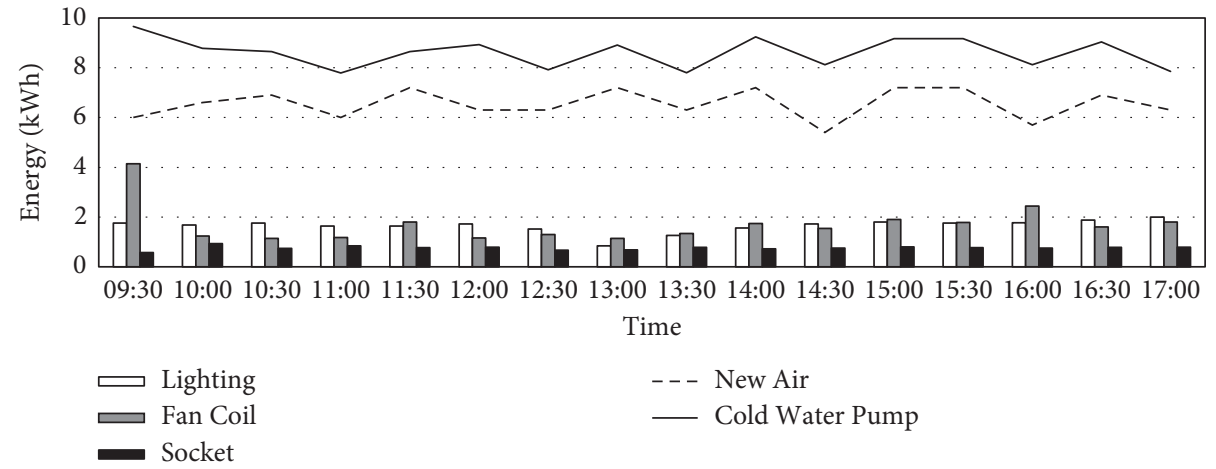

Figure 9: Energy consumption on June 17, 2020.

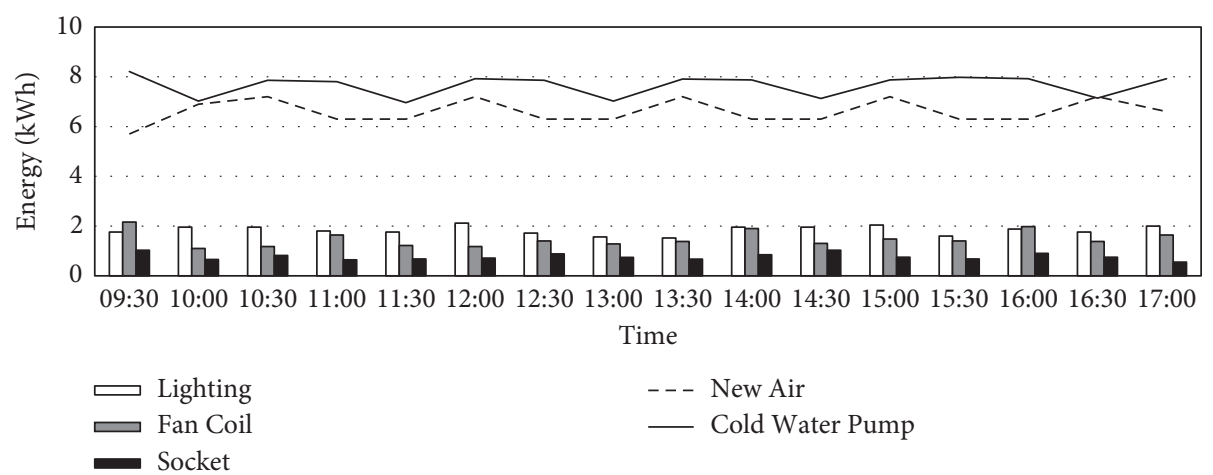

Figure 10: Energy consumption on August 20, 2020.

\section{Conclusions}

Environmental changes in green buildings lead to the need for continuous adjustment of energy-saving control of building systems. Based on the theory of grey incidence, we studied the adaptive adjustment strategies of the decision center in the case of the uncertainty and small sample of the sensing layer in green buildings. At the same time, building subsystems, such as HVAC and lighting subsystems, are often in the grey state mentioned above, and the corresponding adjustment scheme is lacking. We built the Internet of Things platform and developed a data evaluation module in the data center that adapts the input data. Using the "entropy right" calculation, we propose a new grey index correlation algorithm, which proves the parallelism, consistency, order, and convergence. The additional H-FBPNN algorithm is developed in the data center and is applied to the configuration optimization adjustment of HVAC and lighting subsystems. According to the experimental results, the subsystem of the additional H-FBPNN algorithm obviously reduces the system fluctuation and saves more than $30 \%$ of energy consumption without retraining the network. However, the practical application of IoT technology in green buildings should also take into account its reliability and security. On the one hand, the system's fault diagnosis, when the network node failure, can be quickly located and diagnosed, and there are alternative solutions. In addition, we should consider the security of network data; to prevent the theft of information is not only the key issue, but also the direction of future work.

\section{Data Availability}

The training data used to support the findings of this study have not been made available because privacy protection is required by the enterprise where the experiment is conducted.

\section{Conflicts of Interest}

The authors declare that they have no conflicts of interest.

\section{Acknowledgments}

This work was supported by the National Natural Science Foundation of China (61802353) and the Key Specialized Research and Development Breakthrough Program in Henan Province (212102210407).

\section{References}

[1] J. G. Cedeño-Laurent, A. Williams, P. Macnaughton et al., "Building evidence for health: green buildings, current science, and future challenges," Journal of Annual Review of Public Health, vol. 39, no. 4, pp. 156-163, 2018.

[2] D. Minoli, K. Sohraby, and B. Occhiogrosso, "IoT considerations, requirements, and architectures for smart buildings- 
energy optimization and next-generation building management systems," IEEE Internet of Things Journal, vol. 4, no. 1, pp. 269-283, 2017.

[3] H. Kazmi, F. Mehmood, and M. Amayri, "Smart home futures: algorithmic challenges and opportunities," in Proceedings of the 14th International Symposium on Pervasive Systems, Algorithms and Networks, pp. 441-448, UK, June 2017.

[4] W. Tushar, N. Wijerathne, W.-T. Li et al., "Internet of Things for green building management: disruptive innovations through low-cost sensor technology and artificial intelligence," IEEE Signal Processing Magazine, vol. 35, no. 5, pp. 100-110, 2018.

[5] P. J. Werbos, "Computational intelligence for the smart gridhistory, challenges, and opportunities," IEEE Computational Intelligence Magazine, vol. 6, no. 3, pp. 14-21, 2011.

[6] N. Henderson, G. Srivastava, and V. Mago, "A scalable platform to collect, store, visualize, and analyze big data in real time," IEEE Transactions on Computational Social Systems, vol. 8, no. 1, pp. 1-10, 2020.

[7] N. Ayoub, F. Musharavati, S. Pokharel, and H. A. Gabbar, "ANN model for energy demand and supply forecasting in a hybrid energy supply system," in Proceedings of the 2018 IEEE International Conference on Smart Energy Grid Engineering (SEGE), pp. 25-30, Oshawa, Canada, August 2018.

[8] B. M. Wilamowski, "Neural network architectures and learning algorithms," IEEE Industrial Electronics Magazine, vol. 3, no. 43, pp. 56-63, 2009.

[9] D. Tran and Y. K. Tan, "Sensorless illumination control of a networked LED-lighting system using feedforward neural network," IEEE Transactions on Industrial Electronics, vol. 61, no. 4, pp. 2113-2121, 2014.

[10] V. Gorbunov, D. Bobrikov, and A. Balashov, "Information system for room's technological processes management based on fuzzy logic," in Proceedings of the 2016 IEEE NW Russia Young Researchers in Electrical and Electronic Engineering Conference (EIConRusNW), pp. 203-205, St. Petersburg, Russia, 2016.

[11] D. Wijayasekara, O. Linda, M. Manic, and C. Rieger, "Mining building energy management system data using fuzzy anomaly detection and linguistic descriptions," IEEE Transactions on Industrial Informatics, vol. 10, no. 3, pp. 1829-1840, 2014.

[12] O. Linda, D. Wijayasekara, M. Manic, and C. Rieger, "Computational intelligence based anomaly detection for building energy management systems," in Proceedings of the International Symposium on Resilient Control Systems (ISRCS), pp. 77-82, Lake City, UT, USA, August 2012.

[13] S. S. Rawat, V. A. Polavarapu, V. Kumar, E. Aruna, and V. Sumathi, "Anomaly detection in smart grid using rough set theory and $\mathrm{K}$ cross validation," in Proceedings of the International Conference on Circuit, Power and Computing Technologies (ICCPCT), pp. 479-483, Nagercoil, India, March 2014.

[14] S. S. Rawat, S. S. Roy, J. Arora, and G. Suman Shashank, "Multicriteria decision examination in wireless sensor networks," in Proceedings of the International Conference on Optimization, Reliabilty, and Information Technology (ICROIT), pp. 235-239, Faridabad, India, Febuary 2014.

[15] A. Khanna, S. Arora, A. Chhabra, and K. K. Bhardwaj, "I IoT architecture for preventive energy conservation of smart buildings," in Energy Conservation for IoT De-vices. Studies in Systems, Decision and Control, M. Mittal, S. Tanwar,
B. Agarwal, and L. Goyal, Eds., Vol. 206, Springer, Singapore, 2019.

[16] C. K. Metallidou, K. E. Psannis, and E. A. Egyptiadou, "Energy efficiency in smart buildings: IoT approaches," IEEE Access, vol. 99, no. 2, pp. 1-10, 2020.

[17] I. Ivan, T. Roman, K. Natalia, and Z. Khrystyna, "Recovery of incomplete IoT sensed data using high-performance extended-input neural-like structure," Procedia Computer Science, vol. 160, no. 8, pp. 521-526, 2019.

[18] A. S. Ahmad, M. Y. Hassan, M. P. Abdullah et al., "A review on applications of ANN and SVM for building electrical energy consumption forecasting," Renewable and Sustainable Energy Reviews, vol. 33, no. 2, pp. 102-109, 2014.

[19] J. Józefczyk, S. Liu, and R. Vallée, "Emergence and development of grey systems theory," Kybernetes, vol. 38, no. 8, pp. 1246-1256, 2009.

[20] A. Amanna, M. J. Price, and R. Thamvichai, "Grey systems theory applications to wireless communications," Analog Integrated Circuits and Signal Processing, vol. 69, no. 2, pp. 259-269, 2011.

[21] S. Goyal and S. Grover, "Applying fuzzy grey relational analysis for ranking the advanced manufacturing systems," Grey Systems: Theory and Application, vol. 2, no. 2, pp. 284-298, 2012.

[22] S. Liu, C. Lin, and Y. Yang, "Several problems need to be studied in grey system theory," in Proceedings of the 2017 International Conference on Grey Systems and Intelligent Services (GSIS), pp. 1-4, Stockholm, Sweden, August 2017.

[23] Z. Liu and F. Xiao, "An evidential aggregation method of intuitionistic fuzzy sets based on belief entropy," IEEE Access, vol. 7, no. 7, pp. 68905-68916, 2019.

[24] H. Liu, W. Wang, and Q. Zhang, Grey Systems: Theory and Application, Springer, vol. 4883, no. 4, , pp. 44-45, Berlin, Germany, 2011. 